\title{
Producción de tubérculos-semilla de papa con pequeños agricultores en la región de Potosí-Bolivia
}

\author{
Ricardo Wissar*
}

\section{RESUMEN}

Se describe la experiencia en la producción de tubérculos-semilla de papa en la Región de Potosí-Bolivia, realizada a través de un trabajo coordinado entre 16 Instituciones y liderado por PROSEMPA.

Se demostró que el uso de semilla certificada (SC) incrementó en 54\% los rendimientos frente a la semilla del agricultor (SA), obteniéndose una $\mathrm{TRM}=130 \%$ en 71 ensayos con el cv Sani Imilla.

Los resultados obtenidos permitieron la implementación de 90 parcelas de difusión de uso de semilla, obteniéndose un reemplazo acelerado de la SA por la obtenida en éstas parcelas. Se generó una demanda efectiva por SC y además las parcelas sirven de nexo para conectar el sistema bajo certificación con el tradicional. Se organizaron 10 grupos de agricultores semilleristas, quienes produjeron en la campaña 93-94 el 78\% SC de la región. Los resultados indican que ésta parece ser la vía más efectiva para el establecimiento de un sistema sostenible de producción de semilla.

La experiencia fue apoyada por un programa masivo de capacitación, habiéndose realizado 349 eventos con la asistencia de 7,304 agricultores, de los cuales el $27 \%$ fueron mujeres.

Este proceso de desarrollo sólo pudo ser posible debido al trabajo efectivo de coordinación interinstitucional en el cual existió complementación de acciones entre las instituciones participantes.

Palabras claves adicionales: Tubérculos-semilla, semilla certificada, difusión de tecnología de semillas, parcelas demostrativas, semilleristas

Aceptado para publicación: febrero 6,1995.

* Supervisor Regional PROSEMPA 


\section{SUMMARY}

\section{Production of Potato Seed Tubers with Small Farmers in the Potosí Region of Bolivia}

The experience on the production of potato tuber seed in the Potosí region Bolivia, done by 16 institutions under the leadership of PROSEMPA is described. Field work showed that the use of certified seed (CS) increased yields by $54 \%$ in comparison with farmer's seed (FS). A $130 \%$ marginal return rate (MRR) was obtained in 71 trials with the Sani Imilla cultivar.

Results obtained gave way to the establishment of 90 diffusion plots for the use of improved seed, and FS was quickly replaced by seed produced in these plots, generating an effective demand for CS. Furthermore, these plots served as a link between the seed certifying system and the traditional production system.

Ten groups of seed producing-farmers which produced $87 \%$ of the certified seed in the region were organized during the 1993-94 crop season. Results showed that this system could be the most effective way of establishing a lasting system for the production of potato seed.

Field activities were supported by a massive training program. A total or 349 training events were carried out and attended by 7,304 farmers, $27 \%$ of which were women.

The effective coordination between institutions, in which there was complementation of activities, contributed to the success of this development process.

Additional index words.

Seed tubers, certified seed, seed technology

Diffusion, demonstration plots, seed producers

Uno de los principales gestores del Programa Nacional de la Papa en Bolivia es el Proyecto de Semilla de Papa (PROSEMPA). Los ejecutores del proyecto son el Consejo Nacional de Semillas y la Dirección General de Cooperación Internacional del Gobierno de Holanda, quien a su vez contrató a la Empresa Consultora Euroconsult para que en su representación coadyuve a su ejecución. 
PROSEMPA finalizó su primera fase de ejecución de tres años en septiembre de 1994; teniendo como objetivo general el fortalecimiento de los sistemas de multiplicación y distribución de semilla, buscando promover el uso racional de semilla por el agricultor y fortaleciendo la capacidad de las instituciones que desarrollan actividades en cultivo de papa.

Para la realización de sus actividades PROSEMPA priorizó en esta fase actividades en Cochabamba, Chuquisaca y Potosí. Dentro de cada región se definieron zonas de trabajo en base a la presencia institucional, la importancia del cultivo y el número de productores de papa. En el caso de Potosí fueron seleccionadas 8 zonas en las cuales se cultivan aproximadamente 25,300 ha de papa y existen 22,300 unidades agrícolas familiares.

La metodología de trabajo consistió en demostrar a los agricultores las bondades del uso de semilla de buena calidad, enseñarles a producir su propia semilla, generar demanda y organizar pequeños grupos de agricultores remultiplicadores de semilla.

El presente documento intenta describir la experiencia lograda en Potosí en base al trabajo de coordinación interinstitucional realizado, de manera que pueda servir como reflexión para ejecutar este tipo de actividades en otros lugares.

\section{MATERIALES Y MÉTODOS}

\section{Materiales}

Ubicación y descripción de la Región: La región de Potosí está ubicada en el Sud Oeste de Bolivia y presenta condiciones ecológicas diversas. Está conformada por grandes extensiones planas de puna y salares en la parte occidental y una topografía montañosa con altitudes desde 2000 a 4500 msnm en la parte oriental, en su mayoría lugares aptos para la producción de papa, donde se ubican las zonas de trabajo (Mapa 1).

La población rural está geográficamente dispersa en lugares de difícil acceso, está compuesta mayoritariamente por minifundistas cuya producción está orientada a la subsistencia con pequeños excedentes para el mercado.

En la región se observan dos sistemas de producción de semilla de papa: i) el sistema bajo certificación, el cual está regido por las normas del Consejo Nacional de Semillas y representa aproximadamente el 1\% de la necesidad total de semilla de papa, y ii) el sistema tradicional, conformado por miles de pequeños agricultores quienes generalmente producen su propia semilla. 


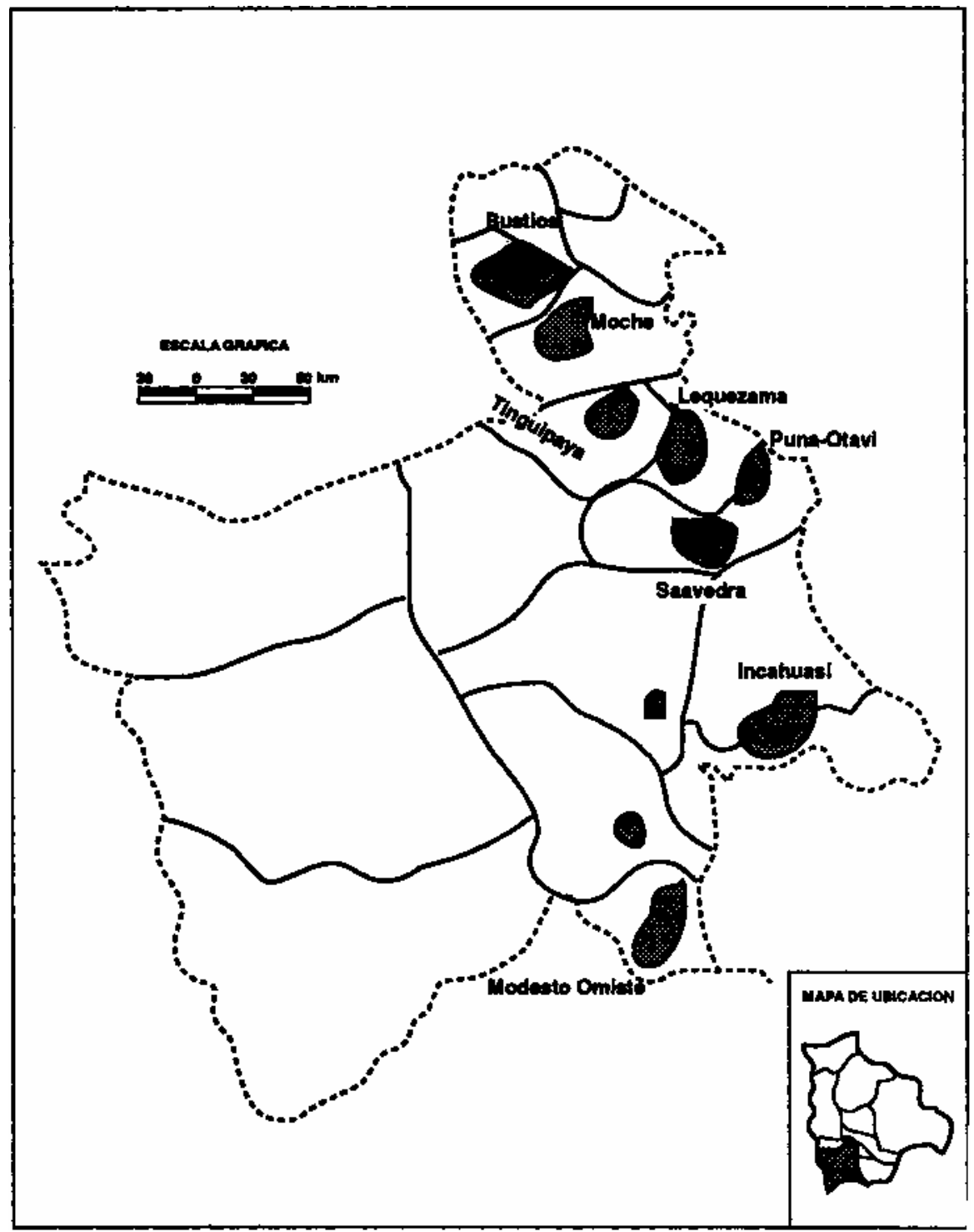

Fig. 1. Zonas de trabajo en la región Potosí

Coordinación Interinstitucional: El trabajo se realizó en coordinación con 16 Instituciones, entre organizaciones no gubernamentales, proyectos de desarrollo y dos Universidades. (Tabla 1). 
Tabla 1. Relación de instituciones co-ejecutoras.

\begin{tabular}{ll}
\hline \multicolumn{1}{c}{ Sigla } & \multicolumn{1}{c}{ Nombre } \\
\hline ACLO & Acción Cultural Loyola \\
CIAC & Centro de Investigación y Apoyo Campesino \\
FH & Fundación contra el Hambre \\
ICADES & Instituto de Capacitación de Desarrollo Social \\
IPTK & Instituto Politécnico Tomás Katari \\
ITSA & Instituto Técnico Superior de Agronomía, Bustillos \\
MINK'A & Proyecto de Desarrollo Agrícola Mink'a \\
ORS-Potosí & Oficina Regional de Semillas de Potosí \\
PAC & Programa de Autodesarrollo Campesino. \\
PCHS & Proyecto Chuquisaca Sud \\
PDACSJO & Proyecto Cotagaita San Juan del Oro \\
PDAI & Programa de Desarrollo Agrícola Integrado \\
PIOXII & Radio Pió XII \\
PRODECIT & Proyecto de Desarrollo Comunal Integral de Tinquipaya. \\
PROSEMPA & Proyecto de Semilla de Papa \\
UATF & Universidad Autónoma Tomás Frías, Potosí \\
UNSXX & Universidad Nacional SigloXX, Llallagua. \\
\hline
\end{tabular}

\section{Métodos}

Las actividades se iniciaron con el establecimiento de las parcelas comparativas de semilla en la campaña 1991 -92, para luego establecer las parcelas de difusión de uso de semilla y culminar con la formación de grupos semilleristas. Todo el proceso fue apoyado por una serie de eventos de capacitación.

Parcelas comparativas de calidad de semilla: Durante la campaña 1991-92 se establecieron 71 parcelas comparativas con el cv Sani Imilla comparando la semilla certificada (SC) procedente de la Unidad de Producción de Semilla de Papa (UPS/ Sepa) frente a la semilla del agricultor (SA). En estas parcelas se utilizó un paquete tecnológico adecuado a las condiciones agro-socioeconómicas de cada zona.

Parcelas de difusión de uso de semilla: Para la campaña 1992-93 se plantaron 52 parcelas de difusión de uso de semilla de primer año (PADUS-1) y 38 de segundo año (PADUS-2). Las PADUS-1 fueron establecidas haciendo uso de 100 - $200 \mathrm{~kg}$ de SC procedentes de UPS/Sepa. Se utilizó el mismo paquete tecnológico de la campaña anterior. Las PADUS-2 se establecieron siguiendo la misma metodología, con la semilla obtenida de PADUS-1.

Estas parcelas tuvieron como finalidad demostrar al agricultor un sistema racional de uso de semilla de buena calidad y producción de su propia semilla, que le permita incrementar sus rendimientos y promover una demanda por semilla de calidad. 
Formación de grupos semilleristas: En base a los resultados obtenidos y experiencias desarrolladas en Colombia (Garay 1992) y en Perú (Franco y Wissar, 1990); PROSEMPA propuso a las Instituciones organizar pequeños grupos de agricultores remultiplicadores para lograr el abastecimiento local de semilla.

La propuesta fue consultada a los agricultores capacitados por el proyecto, quienes decidieron agruparse. Las Instituciones y PROSEMPA asesoraron los procesos de organización, producción y comercialización.

Eventos de capacitación: Todo el proceso fue acompañado de eventos de capacitación. Las instituciones y PROSEMPA utilizaron cursos, talleres, cursillos y días de campo como herramientas de transferencia de tecnología.

Los cursos fueron eventos dirigidos a técnicos y promotores de las diferentes instituciones en aspectos de elaboración de proyectos, organización y producción.

La Unidad de Promoción y Comunicación de PROSEMPA produjo cuatro paquetes educativos que consisten en un conjunto de medios (cartillas, afiches, radiodrama, diaporama y documentos técnicos) en los temas de cosecha y almacenamiento, preparación de suelos y siembra, plagas y enfermedades y producción de semilla. Se realizaron talleres para enseñar el manejo de los paquetes educativos a los técnicos de las instituciones coejecutoras, quienes fueron los encargados de la realización de los cursillos a agricultores con el apoyo del personal de PROSEMPA. La duración de los cursillos fue de medio día y los agricultores recibieron un afiche y cartilla de cada tema.

Los días de campo fueron utilizados para informar, promover e incentivar el uso de innovaciones tecnológicas y fortalecer las relaciones entre agricultores y extensionistas.

\section{RESULTADOS Y DISCUSIÓN}

\section{Parcelas comparativas de calidad de semilla}

Los resultados demostraron que el uso de semilla certificada en el cv Sani Imilla, incrementó los rendimientos en 54\% frente a la semilla del agricultor, obteniéndose una tasa de retorno marginal (TRM) de 130\% (Figura 1; PROSEMPA 1992). Estos resultados son similares a los obtenidos por el Programa de Investigación de Papa en Bolivia (PROINPA) en la campaña 1991-92 con el mismo cultivar en Yamparaes, Chuquisaca (PROINPA, 1993). 


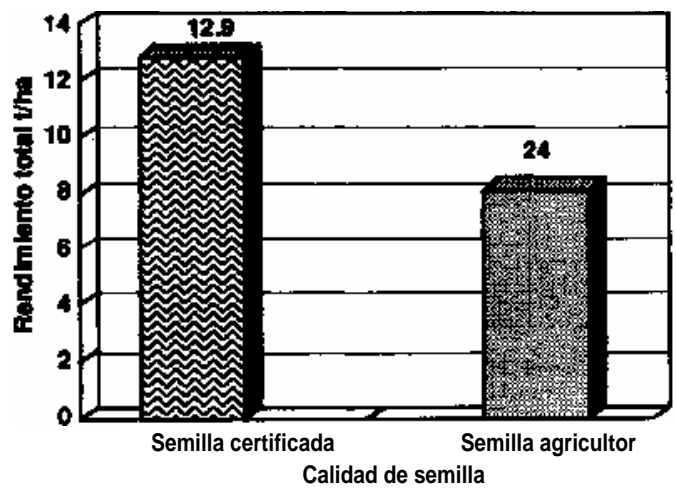

Figura 1. Rendimiento de semilla certificada y semilla del agricultor ent ha, cv Sani Imilla, Potosí, 1991-92.

\footnotetext{
* * Diferencias estadísticas significativas según la prueba de «t»p (0.1)

$\mathrm{n} \quad=$ Número de ensayos

TRM = Tasa de retorno marginal
}

Parcelas de difusión de uso de semilla

Los resultados obtenidos en la campaña 1991-92, permitieron establecer las PADUS-1 y las PADUS-2 cuyos resultados se muestran en la Figura 2.

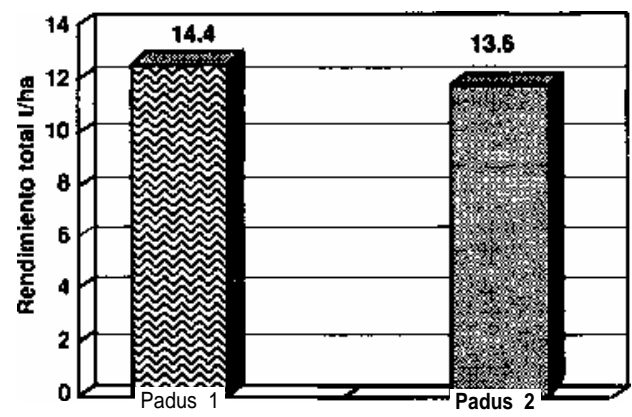

Figura 2. Rendimiento de PADUS-1 y PADUS-2 en gha, cv Sani Imilla,

\section{Potosí 1992-93.}

$\mathrm{ns} \quad=$ No se encontraron diferencias significativas según la prueba de «t»p(0.1)

$\mathrm{n} \quad=$ Número de parcelas

$\mathrm{B} / \mathrm{C}=$ Relación beneficio-costo

Los rendimientos obtenidos entre PADUS-1 y PADUS-2 no fueron estadísticamente diferentes entre sí. Sin embargo, la relación beneficio-costo fue mayor en PADUS-2 por el menor costo de la semilla. Estos resultados coinciden con los obtenidos por González et al. (1994), en Cochabamba utilizando el cv Waych'a. 
Estos trabajos permitieron generar una demanda efectiva por semilla de calidad por parte de los agricultores y se produjo un reemplazo de su propia semilla por la obtenida de las PADUS (PROSEMPA, 1993). Esto demuestra que la semilla de calidad «camina sola» en las manos de los pequeños agricultores.

La razón principal del uso de SC con un paquete tecnológico adecuado a las condiciones del agricultor se debe a que los rendimientos del cultivo en Solivia están entre los más bajos del mundo, pese a ser el cultivo más importante del país. (Kloos y Caero 1992). Posteriormente De Franco y Godoy (1993); al investigar la relevancia de innovaciones tecnológicas en la agricultura boliviana para la economía nacional, encontraron que las innovaciones en el cultivo de papa y luego maíz tienen mayor impacto que las innovaciones en trigo o soya, lo que indica que el uso de un paquete tecnológico adecuado a cada zona es acertado. Se infiere que la masificación de estas actividades ayudarán a incrementar la productividad del cultivo de papa en la región.

\section{Formación de grupos semilleristas}

Características de los grupos: La experiencia en la formación de grupos data de la campaña 1991 -92. En Modesto Omiste, los agricultores beneficiarios de PADUS de la Cooperativa Defensores del Sur implantaron en conjunto sus parcelas en terrenos de la Cooperativa, habiendo sido certificada la producción por la ORS-Potosí. En base a las experiencias logradas en esta zona se formaron otros grupos. (Tabla 2).

Tabla 2. Grupos por zona en la región.

\begin{tabular}{|lllll|}
\hline Zona & \multicolumn{1}{c}{ Nombre } & $\begin{array}{c}\text { Número } \\
\text { Socio }\end{array}$ & $\begin{array}{c}\text { Institución } \\
\text { Co-ejecutora }\end{array}$ & $\begin{array}{c}\text { Año de } \\
\text { formación }\end{array}$ \\
\hline \multirow{3}{*}{ Modesto Omiste } & Coop. Def. del Sur & 16 & PDACSJO & $1991-92$ \\
& La Quesera I & 5 & PDACSJO & $1992-93$ \\
& La Quesera II & 6 & PDACSJO & $1993-94$ \\
& Sagnasti I & 8 & PDACSJO & $1992-93$ \\
& Sagnasti II & 6 & PDACSJO & $1992-93$ \\
Incahuasi & Miraflores & 8 & PCHS & $1993-94$ \\
& Chunchuli I & 8 & PCHS & $1993-94$ \\
& Chunchuli II & 8 & PCHS & $1993-94$ \\
Saavedra & Lagunillas & 8 & MINK'A & $1993-94$ \\
& Chustuco & 25 & CIAC & $1993-94$ \\
Lequezana & Rumiwiñaska & 8 & PDAI & $1993-94$ \\
Puna-Otavi & Hornos & 8 & - & $1993-94$ \\
\hline
\end{tabular}


La ubicación de los grupos fue realizada haciendo un balance acerca de la decisión de los agricultores, lugares tradicionalmente productores de semilla, condiciones agroclimáticas favorables, sanidad de los suelos, accesibilidad, mercado potencial, apoyo institucional y la organización existente. Como no existen lugares que reúnan todos los factores en condiciones óptimas, entonces la ubicación de los grupos fue realizada haciendo un balance de estos factores. Los aspectos débiles de cada grupo se fortalecerán conforme estos se vayan consolidando, lo cual coincide con las apreciaciones de Garay, (1993a).

La Tabla 3 presenta la forma de producción y comercialización por parte de los grupos, como se puede ver no existen esquemas organizacionales únicos debido a la diversidad de la región. Es importante respetar las organizaciones existentes y en todo caso es necesario contar con la aprobación del sindicato, cooperativa o cualquier otra forma organizativa existente para emprender la formación de los grupos.

Tabla 3. Forma de producción y comercialización de los grupos semilleros.

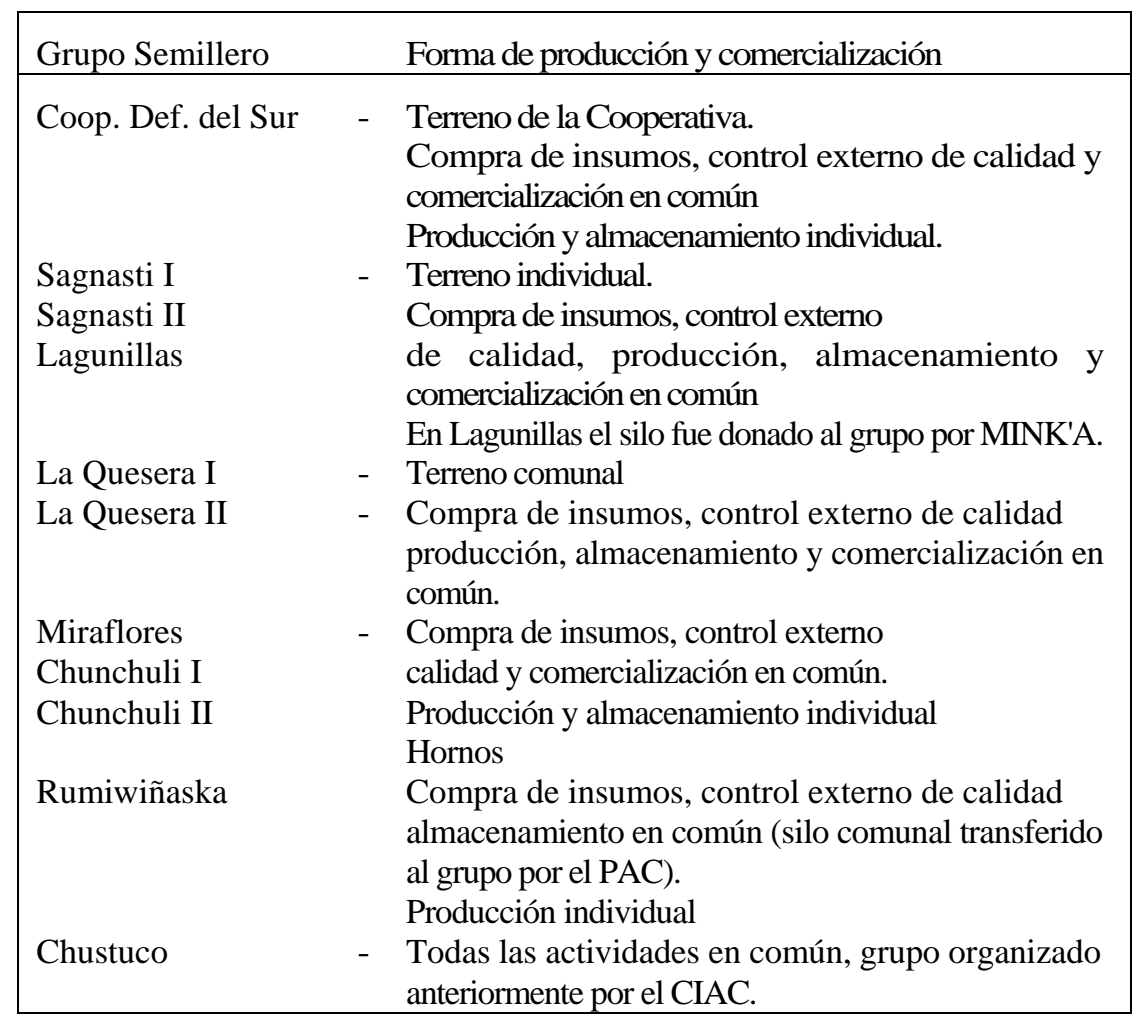


En el caso de la Cooperativa Defensores del Sur, debido al elevado número de socios y por no funcionar eficientemente la producción en común, optaron por realizar la producción individual. En Chustuco participa el 50\% de la comunidad, habiendo sido éste organizado anteriormente por el CIAC para la remultiplicación de semilla de papa, no se observó dificultades en el manejo, debido presumiblemente al constante asesoramiento del CIAC.

La experiencia indica que el número ideal de socios por grupo es de cinco a diez, lo que permite tener grupos homogéneos que facilitan un eficiente desarrollo de actividades. A excepción de la Cooperativa Defensores del Sur y Chustuco que fueron grupos organizados anteriormente, los demás fueron formados expresamente para la producción de semilla.

El control externo de calidad es realizado por la ORS, la que ha aportado fuertemente en el concepto de flujo de semilla y el control interno de calidad, se está logrando progresivamente a través de una permanente capacitación basada en que la mejor propaganda del grupo es la calidad de semilla que produce y comercializa.

La característica principal de la comercialización es la venta en común como producto diferenciado en base a su marca y la etiqueta de certificación. En la zona de Modesto Omiste, gran parte de la semilla producida fue comercializada fuera de la zona debido a mejores precios; consideramos que no es un mercado estable, por lo que se debe tender a establecer mercados más seguros dentro de la zona.

Una característica general en todos los grupos es la compra de insumos en común lo que permite un ahorro y el uso de un paquete tecnológico homogéneo en cada grupo. Hasta el momento la compra de semilla básica y/o registrada para la inyección de los grupos fue canalizada por las instituciones, esta responsabilidad en el futuro deberá ser transferida a los mismos productores.

Únicamente en la zona de Modesto Omiste, los grupos cuentan con una organización sólida al interior de cada uno basada en estatutos y reglamentos que en los demás grupos están en proceso de elaboración. En el caso de M. Omiste la participación activa de los socios en la elaboración de estatutos y reglamentos ha permitido su aplicación.

Finalmente se efectuaron evaluaciones del plan de operaciones de cada campaña, de manera de ir corrigiendo los errores cometidos.

\section{Producción de semilla de papa con grupos de agricultores en la Región}

En la campaña 1993-94 en la Región de Potosí se produjeron 191 t de semilla certificada, de los cuales el $78 \%$ fue producida por los grupos semilleristas (ORS,1994). 
La relación beneficio-costo para esta campaña a excepción del grupo Hornos fueron apreciables (Tabla 4) Un estudio realizado por PDACSJO y PROSEMPA analizaron la rentabilidad del Grupo Semillero Defensores del Sur indicando que ésta es variable a través de los años de producción. En años buenos la relación beneficio-costo fue de 3.5 y en años regulares indica poca ganancia o pérdida. (PDACSJO, 1994).

Tabla 4. Producción de semilla en t por grupo de semilleristas, 1993-94.

\begin{tabular}{|c|c|c|c|}
\hline Grupo semillero & $\begin{array}{c}\text { Área sembrada } \\
\text { (ha) }\end{array}$ & $\begin{array}{c}\text { Producción } \\
\text { Semilla (t) }\end{array}$ & $\begin{array}{l}\text { Relación } \\
\frac{\text { IT-CT }^{*}}{\mathrm{CT}}\end{array}$ \\
\hline Coop. Def. del Sud & 15.45 & 90.18 & 0.97 \\
\hline Sagnasti I & 1.24 & 5.81 & 0.75 \\
\hline Sagnasti II & 1.17 & 6.32 & 0.65 \\
\hline La Quesera I & 0.87 & 7.50 & 0.95 \\
\hline La Quesera II & 0.36 & 2.35 & $0 . \%$ \\
\hline Miraflores & 1.05 & 15.25 & 2.50 \\
\hline Chunchuli I & 1.05 & 8.35 & 1.92 \\
\hline Chunchuli II & 0.80 & 3.75 & 1.40 \\
\hline Chustuco & 0.66 & 5.48 & 1.06 \\
\hline Hornos & 1.66 & 3.80 & -0.42 \\
\hline SUBTOTAL & 24.31 & 148.79 & \\
\hline Lagunillas $\quad * *$ & 0.66 & 6.58 & 1.22 \\
\hline Rumiwiñaska ** & 1.50 & 6.70 & 1.10 \\
\hline SUBTOTAL & 2.16 & 13.28 & \\
\hline TOTAL & 26.47 & 162.07 & \\
\hline
\end{tabular}

Se estableció la noción de flujo, en la que los grupos de agricultores semilleristas demandan semilla al sistema bajo certificación y estos a su vez abastecen de semilla al sistema tradicional. Para la campaña 1993-94 los grupos adquirieron 13.8 t de semilla de las categorías básica y registrada.

Los cinco grupos y 11 productores individuales de semilla de Modesto Omiste formaron la Asociación de Productores de Semilla de Papa (APROSEPA), como una institución coordinadora de las actividades en la zona y de prestación de servicios a los socios. De esta manera se viene desarrollando una red de multiplicadores de semilla, que permitirá la perdurabilidad de los grupos. 
Con el objeto de comercializar el producto y generar demanda por semilla se establecieron las parcelas de promoción, difusión y uso de semilla (PADUS) producida por los grupos en las zonas de demanda.

En tres localidades de Modesto Omiste, donde se realiza siembra temprana se establecieron 20 parcelas para promocionar la semilla, obteniéndose rendimientos promedios de $14 \mathrm{t} / \mathrm{ha}$ que duplicaron los rendimientos de la zona. Esta actividad permitió ligar a los productores de semilla con sus clientes y demostrar la calidad del producto a comercializarse.

El trabajo mancomunado de las instituciones y PROSEMPA está permitiendo el desarrollo de los grupos semilleros y la utilización más eficiente de la capacidad técnica en la Región. Instituciones con experiencia en organización, comercialización, producción y otros aspectos, se conjugan fácilmente para lograr un sistema sostenible de abastecimiento de semillas.

Los mayores problemas que se confrontaron en la formación de grupos se refieren a aspectos de organización y administración. La elaboración de los planes de producción y comercialización con la participación de los socios fue una de las tareas más dificultosas. Consideramos que la elaboración de estatutos y reglamentos es otra actividad indispensable en la formación de los grupos. Las actividades de planificación no son manejadas muy bien por los técnicos, por tanto será necesario continuar con la capacitación en éstos aspectos.

Otro problema fue la presencia del nematodo del quiste en los grupos de Lagunillas y Rumiwiñaska, por lo que la ORS rechazó la semilla producida en éstos campos; sin embargo, los agricultores sostienen que vendieron como semilla la producción en la zona. Lanza, et al (1994) indica que la zona está fuertemente infestada por este nematodo.

Es necesario incidir en la di versificación de cultivos, los grupos deberán producir semilla de otros rubros que permitan contar con agricultores especializados en producción de semillas en general, logrando su fortalecimiento y el manejo racional de los recursos naturales. Esto no es excluyente a que se dediquen a la producción comercial en cualquier rubro.

Una buena producción de semilla no representa automáticamente un mejoramiento en los niveles de ingreso de los productores, puesto que es a través de la comercialización que la mayor producción se convierte en ingresos; por lo tanto el apoyo a los grupos en la comercialización continúa siendo necesario. 
Los resultados obtenidos permiten inferir que el establecimiento de esquemas de producción y distribución de semilla de papa con pequeños agricultores a través de grupos organizados, parece constituir una de las vías más efectivas para lograr un sistema perdurable en la Región. Estos resultados coinciden con las apreciaciones de Franco y Wissar (1090), Montealegre y Alvarez, (1992) y Garay en (1992) y (1993).

El sistema de producción y distribución de semilla de papa en la Región: Con las instituciones que realizan acciones en el cultivo, se conformó el Comité Interinstitucional de la Papa (CIPA), como un ente de asesoramiento al Consejo Regional de Semilla. Al interior del CIPA se establecieron y definieron responsabilidades en el sistema de abastecimiento y uso semilla para la Región.

Se establecieron cuatro niveles en la producción y uso de semilla de calidad. El nivel 1 dedicado a la producción de semilla pre-básica, el nivel 2 a semilla básica y registrada, el nivel 3 remultiplica la semilla obteniendo semilla certificada y fiscalizada que constituyen el sistema bajo certificación. En el nivel 4 están los usuarios de semilla. PROSEMPA (1992)

La promoción del uso de semilla en el último nivel a través de las PADUS, está permitiendo generar una demanda por semilla de abajo hacia arriba, conectando el sistema bajo certificación con el tradicional.

\section{Eventos de Capacitación}

La realización de 230 cursillos y 119 días de campo a agricultores en las zonas de trabajo fue el soporte en que se basó la construcción del modelo planteado.

En los cursillos participaron 7,304 agricultores, de los cuales 27\% fueron mujeres. A los días de campo asistieron 5,188 agricultores con un porcentaje de participación de mujeres similar al de los cursillos. Se empezó a valorar la participación de la mujer en el proceso productivo y su importancia en la toma de decisiones al interior del núcleo familiar para la adopción de una nueva tecnología.

La realización de cinco cursos sobre aspectos de formulación de proyectos, organización y producción, además de los 11 talleres de capacitación sobre el uso de paquetes educativos permitió elevar el nivel de conocimientos de los técnicos sobre la problemática papera.

El uso de los paquetes educativos se constituyó un instrumento novedoso y motivador para los técnicos de las instituciones, quienes replicaron numerosos cursillos en sus áreas de trabajo. 
La ejecución de los eventos de capacitación facilitaron los procesos de coordinación institucional, sin la cual no hubiera sido posible llegar con una presencia significativa a los agricultores de la región.

\section{CONCLUSIONES}

- Se demostró que el uso de SC incrementó los rendimientos en 54\% frente a la SA, con una tasa de retorno marginal de $130 \%$ en 71 comparaciones realizadas con el cv Sani Imilla.

- Las parcelas de difusión uso de semilla permitieron un reemplazo de la semilla del agricultor por la generada en éstas parcelas y la utilización de un paquete tecnológico adecuado a cada zona.

- Las parcelas de difusión de uso de semilla están permitiendo generar una demanda efectiva de semilla y servir de nexo para ligar el sistema bajo certificación con el sistema tradicional.

- $\quad$ El establecimiento de esquemas de producción y distribución de semilla de papa a través de pequeños agricultores organizados parece ser la vía más efectiva para establecer un sistema sostenible en Bolivia. En la campaña 199394 el 78\% de la semilla certificada en la Región de Potosí fue producida por 10 grupos semilleros.

- Se estableció un sistema de producción y distribución de semilla de papa para la región, a través del Comité Interinstitucional de la Papa.

- La capacitación masiva de agricultores a través de los cursillos y días de campo fue el soporte en que se basó la estrategia de trabajo.

- $\quad$ Este proceso de desarrollo sólo pudo ser posible con la realización de un trabajo de coordinación interinstitucional efectivo, en el cual existió una complementación de acciones entre las instituciones que desarrollan actividades en el cultivo.

\section{REFERENCIAS BIBLIOGRÁFICAS}

1. De Franco, M.; Goday,R. 1993.Potatoes-ledgrowth: the macro-económic effects of technological innovation in Bolivian Agriculture. The Journal of Developments Studies, vol 29. 
2. Franco, E.; Wissar, R. 1990. Sistemas de distribución de semilla de papa: la experiencia peruana. Seminario Taller sobre metodología para la investigación agronómica y socio-económica de la producción y distribución de tubérculos-semilla de papa. Quito, Ecuador. 13 p.

3. Garay, Adriel E. 1992. Centro Internacional de Agricultura Tropical. Desarrollo de pequeñas empresas semilleras (PES): Algunas consideraciones importantes. Documento de Trabajo $N^{\circ}$ 104. Cali, Colombia. $13 \mathrm{p}$.

4. Garay, Adriel E. 1993. Desarrollo de un sistema integral de abastecimiento de semilla de papa en Bolivia en base a pequeñas empresas remultiplicadoras de semilla (PESEMPAS). Informe de Consultoría a PROSEMPA. Cochabamba, Bolivia. 21 p.

5. Garay, Adriel E. 1993 a. Desarrollo de pequeñas empresas multiplicadoras de semilla de papa (PESEMPAS). Informe de Consultoría a PROSEMPA, Cochabamba, Bolivia. 19 p.

6. González, S; Devaux, A.; Fernández - Northcote, E.; Alvarez, V. 1994. Evaluación agroeconómica de la semilla certificada bajo condiciones de producción de agricultores, p. 27-28. En: III Reunión Nacional de la Papa, Compendio Exposiciones Cochabamba, Bolivia.

7. Kloos, J.P; Caero, G. 1992. Sistemas de producción de semilla de papa. p. 30-41. En: Memorias II Reunión Nacional de la Papa. Cochabamba, Bolivia.

8. Lanza, M.E.; Wissar, R.; Montesinos, R.; Vacaflor, G.; Arce, M. 1994 Distribución de N.aberrans y Globodera spp en el Departamento de Potosí p. 125-126. En: III Reunión Nacional de la Papa comprendido de Exposiciones. Cochabamba, Bolivia.

9. Montealegre, A.; Alvarez, R. 1992. Organización e implementación de pequeñas empresas semilleras (PES). Informe de Consultoría a PROSEMPA, Cochabamba, Bolivia.

10. Oficina Regional de Semillas Potosí, 1994. Informe Campaña 1993-94, Potosí, Bolivia.

11. Programa de Investigación de la Papa - PROINPA. 1993 Informe Anual. Compendio 1992-1993, p. 47 Cochabamba, Bolivia.

12. Proyecto de Desarrollo Agropecuario Cotagaita San Juan del Oro y Proyecto Nacional de Semilla de Papa. 1994. Defensores del Sur, experiencias con grupo de productores de semilla de papa. 23 p. Cochabamba, Bolivia. 
13. Proyecto de fortalecimiento del sistema de multiplicación y distribución de semilla de papa en Solivia PROSEMPA. 1993. Informe 199192.Cochabamba, Bolivia, 28p.

14. Proyecto de fortalecimiento del sistema de multiplicación y distribución de semilla de papa en Bolivia PROSEMPA. Regional Potosí. 1993. Resultados de la gestión 1992-93. Potosi, Bolivia, 83 p.

15. Proyecto de Fortalecimiento del Sistema de Multiplicación y Distribución de Semillade Papa en Bolivia. PROSEMPA Regional Potosí, 1994. Resultados de la gestión 1992-93 Potosí, Bolivia 127p.

\section{AGRADECIMIENTO}

El autor agradece la colaboración recibida por Directores y Técnicos de las instituciones co-ejecutoras, los Coordinadores Zonales de la Regional Potosí y el apoyo recibido de la Dirección de PROSEMPA en la ejecución de este trabajo. 